\title{
Impact of shared and autonomous vehicles on travel behavior
}

\author{
Naveen Eluru $^{1}$ (D) . Charisma F. Choudhury ${ }^{2}$
}

Published online: 25 October 2019

(c) Springer Science+Business Media, LLC, part of Springer Nature 2019

The mobility landscape is undergoing a radical transformation with the advent of shared and autonomous vehicles (AVs). These new forms of mobility are leading to marked changes in individual behavior (such as travel mode choice, activity patterns and destination choices), household choices (such as residential location and vehicle ownership) and system level decisions (such as new infrastructure needs and safety legislation). A good understanding of how the shared and autonomous vehicles are impacting the individual, household and system level decisions would allow academics, industry professionals and policy makers to make informed decisions for improving the transportation system.

The profound impact of shared and autonomous vehicles on travel behavior have led to significant research efforts in these areas in recent years. These are reflected in publication of two previous special issues in Transportation dedicated to this research area in the last 2 years (see Akar and Erhardt 2018; Stathopoulos and Sener 2017). However, the papers in those issues have primarily focused on a single choice in isolation (such as effect of autonomous vehicles on trip generation, and vehicle-miles-travelled). Building on these previous findings, transport researchers are now looking at wider impacts including: (a) how the new mobility options will affect the day-to-day activity patterns, (b) how different behavior models can be combined in simulation tools for predicting the state of the network and (c) most importantly, how the combination of autonomous vehicle technology and ride-sharing and/or mobility-on-demand services will lead to even more radical changes in travel behavior.

The current Transportation Research Board (TRB) special issue of Transportation presents eight research articles contributing to the literature on some of these new research questions and challenges by employing innovative and rigorous quantitative approaches. The papers were sourced from articles submitted to the TRB Committee on Travel Behavior and Values (ADB10) and the TRB Committee on Travel Demand Forecasting (ADB40).

Naveen Eluru

Naveen.Eluru@ucf.edu

https://people.cecs.ucf.edu/neluru

Charisma F. Choudhury

C.F.Choudhury@leeds.ac.uk

1 Department of Civil, Environmental and Construction Engineering, University of Central Florida, 12800 Pegasus Drive, Room 301D, Orlando, Florida 32816, USA

2 Institute for Transport Studies and School of Civil Engineering, University of Leeds, Leeds, UK 
The first paper, Xie et al. (2019), presents a discrete choice model framework to capture the sequential but inter-connected decision-making stages specific to on-demand service usage. The framework includes a hybrid choice model for service subscription and three logit mixture models with inter-consumer heterogeneity for the service access, menu product choice and opt-out choice. The methodology is applied to a case study of an innovative personalized on-demand real-time system which incentivizes travelers to select more sustainable travel options. The data for model estimation is collected through a smartphone-based context-aware stated preference survey. The models provide useful insights about the Value of Time (VOT) in presence of the reward system, instrumental in setting the ground for different behavioral scenarios of the proposed novel on-demand system.

The second paper, Wang and Akar (2019), examines individual level safety perceptions of autonomous vehicle interactions with neighborhood environment and roadway infrastructure. The research has been conducted drawing on a large individual level dataset from the four-county Puget Sound region. The authors examine individual responses on the level of their concern with autonomous vehicles on a five-point ordered scale ranging from "not at all concerned" to "Very Concerned" using a generalized ordered logit model. The authors report that young individuals (18-34), current car-share members and individuals with interest in riding autonomous vehicles are less likely to have concerns. On the contrary, non-automobile road users such as public transit riders and pedestrians are more concerned with autonomous vehicle interactions. The findings from the study will provide useful inputs for policy makers and practitioners for identifying potential remedial solutions toward addressing road user concerns associated with autonomous vehicles.

The third paper, Vyas et al. (2019), focuses on modelling the effects of AVs on the regional level by proposing modifications to the Activity-Based travel demand Model (ABM) developed for Columbus, $\mathrm{OH}$, metropolitan region. The model modifications include multiple adjustments to the travel demand sub-models, network assignments, as well as an addition of a new sub-model for vehicle routing and parking that addresses such new phenomenon as empty AV relocation trips. The ABM suite is deployed for evaluating the potential impacts of $\mathrm{AVs}$ on the accessibility measures, activity participation, tour formation, and mode choice and provide useful policy insights.

The fourth, fifth and sixth papers, Vosooghi et al. (2019), Dandl et al. (2019) and SeguiGasco et al. (2019), also use agent-based modelling/simulation suites, but in the context of autonomous ride-hailing/mobility-on-demand. Vosooghi et al. (2019) propose an integrated framework that accommodates individual level preferences for ride hailing in modeling a metropolitan region level decision processes. Specifically, they embed user trust and willingness to use autonomous vehicles' services within an activity based simulator (MATSim) to evaluate the influence of autonomous vehicle fleet sizes in the metropolitan region of Rouen-Normandie, France. The data for the user level preferences have been obtained from a local survey and are used to update the embedded activity scoring function in MATSim. The simulation outputs are evaluated in terms of fleet usage (including corresponding mileage), customer waiting times and time of day demand distribution. The results underscore the importance of socio-demographic differences and individual preferences on the operator fleet size decision processes.

Dandl et al. (2019) propose an agent-based simulation framework to evaluate and quantify the impact of spatio-temporal demand forecast aggregation on the operational efficiency of a Shared Autonomous Mobility Service (SAMS) fleet. This is achieved by combining an offline demand forecasting method with a joint AV-user assignment and empty AV repositioning strategy. Taxi data, from New York is used to simulate the 
operation of a SAMS fleet across various spatio-temporal aggregation levels providing useful insights about the optimum planning strategy.

Segui-Gasco et al. (2019) also propose an agent-based simulation framework for evaluating the impacts of Autonomous Mobility on Demand (AMoD) services. This framework is composed of two main agent-based components: a demand generation and mode shift model (implemented using MATSim) and a fleet simulator for providing a highly realistic operational execution of autonomous and manually-driven transport fleets (IMSim). The model has been deployed in testing alternative scenarios in the Royal Borough of Greenwich, London, UK. A wide range of effects arising from the adoption of AMoD services is assessed in this regard. The case study demonstrates how the framework can be utilized by travelers, service-operators, the city, and vehicle manufacturers to ensure efficient use of AMoD.

The seventh paper, Gao et al. (2019), explores the differences in preferences towards personal AV, shared AV and compare it against usual ride-sharing options. Of particular focus is the influence of autonomous vehicles on travelers' value of time perception. The authors conducted their investigation employing the existing ride hailing services as a potential analogy for autonomous vehicles. Specifically, using a Stated Preference data elicitation approach, individuals were presented with alternatives contrasting using a personal vehicle versus adopting a ride hailing service. Randomly selected respondents were informed either that their ride hailing service is driverless or their ride hailing service is equipped for multi-tasking. In the former case, the estimated value of time was higher than the traditional ride hailing service indicating some apprehension among individuals toward fully autonomous vehicles. On the other hand, in the latter case, the estimated value of time was about $50 \%$ lower than personally driving. Overall, the results highlight the inherent contradictions among individuals in their potential adoption of autonomous technology.

The last paper, Cai et al. (2019) also investigates travel behavior in the context of autonomous Mobility on Demand (MOD), but the special focus is on the first mile travel with an emphasis on public acceptance and service design. A stated preference survey was conducted in Singapore separately for drivers and current public transit users. The driver branch of the survey provides automated vehicle driven MOD alternatives (premium, economy and sharing) or existing bus transit as alternatives to current driving scenario. The public transit branch of the survey elicits traveler responses for MOD alternatives and existing alternatives (public bus, bike and walk). The SP data was analyzed using the logit kernel model structure that allows for parameters to vary across travelers. The model results indicate that about $31 \%$ of current transit riders and $57 \%$ of current drivers are willing to adopt autonomous vehicle based MOD alternatives. The study also identified significant influence of socio-demographics affecting the preference for premium, economy and sharing MOD alternatives.

The papers, with data from seven different countries, encompass a wide range of behavior models and simulation techniques and contribute to a smoother transition to the new mobility landscape. We therefore believe they will be immensely useful to both the academics and practitioners. 


\section{References}

Stathopoulos, A., Sener, I.N.: Transforming mobility systems with sharing and automation. Transportation 44(6), 1255-1259 (2017)

Akar, G., Erhardt, G.D.: User response to autonomous vehicles and emerging mobility systems. Transportation 45(6), 1603-1605 (2018)

Publisher's Note Springer Nature remains neutral with regard to jurisdictional claims in published maps and institutional affiliations. 